\title{
Prédiction et rétrodiction
}

Claude Grignon

\section{OpenEdition}

\section{Journals}

Édition électronique

URL : http://journals.openedition.org/ress/135

DOI : $10.4000 /$ ress. 135

ISSN : 1663-4446

\section{Éditeur}

Librairie Droz

\section{Édition imprimée}

Date de publication : 1 septembre 2008

Pagination : 75-90

ISBN : 978-2-600-01242-3

ISSN : 0048-8046

Référence électronique

Claude Grignon, "Prédiction et rétrodiction », Revue européenne des sciences sociales [En ligne], XLVI-142 | 2008, mis en ligne le 01 septembre 2011, consulté le 19 avril 2019. URL : http:// journals.openedition.org/ress/135; DOI : 10.4000/ress.135 


\section{CHAPITRE IV}

\section{PRÉDICTION ET RÉTRODICTION}

\section{L'ÉTUDE STATISTIQUE}

Rapprocher, regrouper, c'est déjà préférer la relation à l'objet, à l'événement concrets, c'est-à-dire singuliers; en quoi la généralisation statistique se distinguet-elle de la généralisation descriptive à laquelle on parvient en partant de l'observation? Offre-t-elle par rapport à celle-ci des avantages particuliers?

L'étude statistique est le degré supérieur d'une quantification des objets qui, même à un niveau rudimentaire, rend leur représentation plus exacte, plus précise, plus impersonnelle, moins dépendante de la subjectivité de l'observateur. Une mesure est une convention, mais une convention qui n'a de sens que dans son application à des objets qu'elle contribue à définir, et dont les résultats s'imposent à tous. Un croquis coté laisse moins de place à la fantaisie de l'ethnographe et à l'imagination de ses destinataires qu'un dessin d'art; le récit le plus littéraire gagne en réalisme lorsqu'on y introduit des notations chiffrées ( Comme il faisait une chaleur de 35 degrés, le boulevard Bourdon était absolument désert»). On peut sans doute mesurer des artefacts et des illusions d'optique; mais la mesure limite le risque interprétatif en soumettant les constats à un contrôle collectif impersonnel. L'étude statistique contrarie la sociologie littéraire; elle lui oppose une orientation et une culture divergentes, un outillage nouveau, une manière différente de penser et d'écrire. Au goût pour la variété, qui s'attache à la diversité mais aussi à la singularité des objets et des événements, elle substitue la recherche systématique de la variation (Bachelard 1980: 30) ${ }^{1}$. Le développement de la statistique résulte de son application aux problèmes de subsistance et de distribution des ressources rares; la loi des grands nombres s'est appliquée d'abord au grand nombre, aux pauvres inconnus et anonymes des villes. La notion d'individu anonyme et interchangeable va de pair avec le caractère impersonnel des enquêtes; elle s'impose aussi bien à ceux à qui celles-ci sont administrées qu'à ceux qui les conçoivent et qui les réalisent. Elle s'oppose à la conception littéraire

1 «La pensée préscientifique ne s'acharne pas à l'étude d'un phénomène bien circonscrit. Elle cherche non pas la variation, mais la variété. Et c'est là un trait particulièrement caractéristique: la recherche de la variété entraîne l'esprit d'un objet à l'autre, sans méthode; l'esprit ne vise alors que l'extension des concepts; la recherche de la variation s'attache à un phénomène particulier, elle essaie d'en objectiver toutes les variables, d'éprouver la sensibilité des variables. Elle enrichit la compréhension du concept et prépare la mathématisation de l'expérience». G. Bachelard, La formation de l'esprit scientifique, Paris, Vrin, 1980, p. 30. 
de l'individu unique et irremplaçable (comme l'œuvre d'art), qui s'applique aussi bien aux personnages des romans qu'au romancier et au lecteur. Elle va aussi de pair avec la mécanique universelle du calcul; même au niveau élémentaire de la statistique descriptive, la mathématisation force à plus de rigueur dans le raisonnement, limite l'exploitation abusive des possibilités offertes par la souplesse et par la puissance d'évocation du langage naturel, interdit les effets rhétoriques qui visent à émouvoir le lecteur pour le convaincre. C'est sans doute pourquoi les sociologues les plus littéraires, réfractaires à la «froideur» et la «sécheresse » des chiffres, et plus encore à l'informatique, s'en méfient et la rejettent, et, quitte à réduire la sociologie à l'ethnographie, campent sur l'opposition entre qualitatif et quantitatif.

Ces gains réels en objectivité entretiennent l'illusion selon laquelle les méthodes quantitatives seraient à tous égards et dans tous les cas plus objectives, y compris au niveau de la conceptualisation et du réalisme des abstractions, que les méthodes d'observation en langage naturel; cette réputation d'objectivité et de scientificité supérieures a conduit depuis longtemps les pseudo-théories et les systèmes de pensée à s'appuyer sur les données statistiques qui leur conviennent. On retrouve la confusion, classique, entre l'exactitude et la vérité (dont témoigne l'assimilation des «sciences dures» aux «sciences exactes»), confusion entretenue par l'ambiguïté du «juste», commun au calcul et à la morale, à mi-chemin entre l'exact et le vrai et participant des deux; l'ubiquité de la balance, qui passe du marché au tribunal, symbolise le mélange des idées et des valeurs d'objectivité, d'impartialité et d'équité. On oublie par là que si l'exactitude est bien une condition nécessaire de la vérité, elle n'en est pas la condition suffisante ${ }^{2}$. Soumettre les faits sociaux (ou historiques) à la mesure est sans doute la manière la plus radicale de les naturaliser; mais à la différence du physicien ou du biologiste expérimentateur, le sociologue, l'économiste ou l'historien statisticiens ne peuvent compter sur la réalité matérielle pour tester la pertinence des abstractions qu'ils mesurent; la résistance qui s'oppose à leur subjectivité est celle du langage et du raisonnement mathématiques, et non celle des choses. Dans l'expérimentation des sciences de la nature, «l'abstraction mauvaise, sans correspondance suffisante avec la réalité, sans fondement objectif, s'avère le plus souvent aussitôt telle par une évidence physique, matérielle; en recherche statistique, au contraire, des chiffres comme tels ne refusent jamais d'être combinés avec d'autres chiffres, la correspondance ou la non-correspondance avec quelque réalité objective n'est pas (...) un fait qui, comme on dit 'saute aux yeux'» (Simiand 1922: 22) .

L'étude statistique n'empêche donc pas l'auto-immunisation des théories et des doctrines. Qu'il s'agisse du choix et de la construction des variables, de la rubrication (le choix des noms qu'on donne aux variables et aux catégories qui les

2 Oublier à l'inverse que l'exactitude est la condition nécessaire de la vérité ramène en deçà de la science, à la pensée spéculative. C'est ainsi, par exemple, que Renan oppose « la recherche et la discussion des faits, sans autre souci que l'exactitude», empreinte de «platitude et d'insignifiance» et «le sens historique » qui se manifeste « comme par une sorte de révélation», «la vérité générale du récit»; d'un côté la «vieille école des bénédictins », la lettre, de l'autre l'esprit, «la gloire souveraine d'inventer», «le génie créateur», la «grande école» qui «est arrivée à l'histoire par la politique». Essais de morale et de critique, Paris, Michel Lévy, 1859, pp. 115, 132, 133.

3 F. Simiand, Statistique et expérience, op. cit., pp. 22, 23. 
composent et qui désignent la réalité à laquelle elles sont censées correspondre), de la sélection des relations au moyen du plan de tris, ou de l'interprétation des résultats et de leur transcription en langage naturel, les opérations dont dépendent le réalisme des découpages et la pertinence des concepts sont des opérations de l'esprit, qui peuvent être, et ne sont que trop souvent inspirées, influencées, voire dictées par des partis-pris spéculatifs, par les a priori d'une pseudo-théorie. L'utilisation ad hoc des statistiques est un des traits par lesquels les pseudo-théories contreviennent le plus aux principes de la science, tout en ressemblant le plus à une véritable théorie. Les statistiques servent couramment et depuis longtemps d'ultima ratio dans les affrontements entre doctrines rivales; s'appuyer sur des enquêtes (le plus souvent, il est vrai, de seconde main), exhiber tableaux et graphiques, argumenter «chiffres à l'appui » est un moyen éprouvé de donner une apparence scientifique à un système de pensée et de lui conférer ainsi une autorité et une légitimité capables d'assurer sa supériorité idéologique et politique. $\mathrm{Ce}$ détournement ne peut que renforcer le préjugé commun, selon lequel «avec la statistique on prouve tout ce que l'on veut, ou encore (ce qui n'est qu'une autre forme de la même opinion) qu'avec la statistique on ne prouve rien » (Simiand 1922: 2$)^{4}$.

L'indépendance des catégories et des dénominations par rapport aux partispris des auteurs de théories et des écoles rivales est assurée, en principe, par la division du travail entre les chercheurs et les statisticiens professionnels. En la matière, les règles et les précautions qu'énumère F. Simiand sont toujours valables: «établissement des données élémentaires, sinon toujours par des spécialistes compétents, du moins alors dans des cadres et avec des précautions qui visent à un enregistrement aussi automatique que possible; collection, réunion, élaboration dans des conditions définies et surveillées, suivant des techniques et des méthodes étudiées et déterminées, présentation des résultats avec indication des conditions d'établissement, avec critique et appréciation de leur valeur et, par suite, de leur emploi possible » (Simiand 1922: 25) ${ }^{5}$. Mais en pratique les instituts de statistiques doivent constamment défendre leur indépendance, non seulement vis-à-vis de leurs clients ou du pouvoir politique, mais aussi des écoles de pensée qui divisent les sciences de l'homme. Leur position d'arbitre et de prescripteur en fait l'enjeu de luttes d'influence, chaque école s'efforçant, par sociologue introduit ou par statisticien converti, de faire prévaloir les catégories et les découpages de la réalité qui lui conviennent.

L'étude statistique s'impose aux sciences de l'homme comme instrument de généralisation, mais aussi pour sa capacité de discrimination par les résultats ${ }^{6}$. Dans la mesure où elle oblige à faire des prédictions susceptibles d'être démen-

$4 \quad$ Id., p. 2.

5 Id., p. 25.

6 Le pouvoir de réfutation de l'étude statistique dépend sans doute, là encore, du niveau de généralité de la théorie et surtout de la nature de cette généralité. Ainsi, par exemple, les relations entre l'origine et la position sociales, mesurées à la profession, au niveau de diplôme, au niveau de revenu des enquêtés et de leurs parents, etc., permettent de vérifier une théorie partielle, comme celle de l'héritage social; on a affaire en effet à un concept défini par un ensemble de régularités définissables. Il n'en va plus de même dès lors qu'on entreprend d'élargir la théorie en lui donnant le titre plus ambitieux, plus général mais plus vague de théorie de la reproduction. 
ties, on peut dire qu'elle constitue un équivalent de l'expérimentation; le sociologue, ou l'historien qui demande à l'enquête de confirmer des relations entre variables transforme ses intuitions en hypothèses susceptibles d'être rejetées. Mais l'étude statistique l'incite du même coup à se référer plus ou moins consciemment à la conception de l'explication inhérente aux sciences expérimentales et aux sciences nomothétiques, à admettre qu'il suffit de prédire pour expliquer. Le sociologue statisticien qui raisonne en expérimentateur a aussi de fortes chances de retrouver la conception de la causalité dérivée de la physique classique, qui exclut la pluralité et la rencontre des causes. Le plan de tris qu'il a en tête s'articule autour de l'opposition entre les variables trieuses, explicatives, et les variables triées, à expliquer; si certaines des relations qu'il anticipe sont «de simple coexistence» ou de «concomitance», la plupart d'entre elles, et celles qu'il privilégie, sont de «causation ${ }^{7}$. Dans cette perspective, une analyse des données qui tient ses promesses est celle qui permet, conformément au principe durkheimien, de distinguer entre les «causes profondes», les «vraies causes» et les «contingences», les «causes occasionnelles» (Simiand 1903: 133) ${ }^{8}$, et d'en finir avec «le prétendu axiome de la pluralité des causes» en attribuant à chaque effet une cause unique ${ }^{9}$. Le raisonnement statistique («si...alors») tend par ailleurs à privilégier l'implication logique, « où le facteur temps n'intervient pas »

7 F. Simiand, Statistique et expérience, op. cit., p. 59.

8 Cf. F. Simiand, «Méthode historique et science sociale», art. cit., pp. 133 sq. : «Nous retrouvons là, [à la fin de l'Histoire politique de l'Europe contemporaine de Ch. Seignobos] prise sur le vif, cette disposition d'esprit qui tout à l'heure, dans l'explosion d'un rocher, nous énumérait comme causes le roc, la poudre, le feu et oubliait la cause véritable, la force brisante du gaz. (...) On note l'étincelle, on oublie la puissance d'explosion de la poudre (...). Indiquer la cause occasionnelle d'un fait n'est en aucune façon expliquer. Si donc l'étude des faits humains tend à expliquer, au sens scientifique du mot, elle tendra par là-même, non certes à ignorer l'élément individuel ou contingent, mais à en faire la part, afin, dans ses résultats propres, d'en éliminer l'action: elle se proposera comme sa tâche dominante non pas de mettre en évidence la suite de ces contingences, mais au contraire de dégager les relations stables et définies qui, une fois ces contingences constatées et mises à part, peuvent apparaître entre les phénomènes ».

9 «[Stuart Mill] admet (...) qu'un même conséquent ne résulte pas toujours d'un même antécédent, mais peut-être dû tantôt à une cause et tantôt à une autre. Cette conception du lien causal, en lui enlevant toute détermination, le rend à peu près inaccessible à l'analyse scientifique; car il introduit une telle complication dans l'enchevêtrement des causes et des effets que l'esprit s'y perd sans retour. Si un effet peut dériver de causes différentes, pour savoir ce qui le détermine dans un ensemble de circonstances données, il faudrait que l'expérience se fît dans des conditions d'isolement pratiquement irréalisables, surtout en sociologie. Mais ce prétendu axiome de la pluralité des causes est une négation du principe de causalité (...) Si le rapport qui unit C à A est purement chronologique, il n'est pas exclusif d'un autre rapport du même genre qui unirait C à B par exemple. Mais si, au contraire, le lien causal a quelque chose d'intelligible, il ne saurait être à ce point indéterminé. S'il consiste en un rapport qui résulte de la nature des choses, un même effet ne peut soutenir ce rapport qu'avec une seule cause, car il ne peut exprimer qu'une seule nature. Or il n'y a que les philosophes qui aient jamais mis en doute l'intelligibilité de la relation causale. Pour le savant, elle ne fait pas question; elle est supposée par la méthode de la science. Comment expliquer autrement et le rôle si important de la déduction dans le raisonnement expérimental et le principe fondamental de la proportionnalité entre la cause et l'effet? Si donc on veut employer la méthode comparative d'une manière scientifique, c'est-à-dire en se conformant au principe de causalité tel qu'il se dégage de la science elle-même, on devra prendre pour base des comparaisons que l'on institue la proposition suivante: A un même effet correspond toujours une même cause». E. Durkheim, Les règles de la méthode sociologique, op. cit., p. 126. 
(Fagot-Largeault 1989: 264) ${ }^{10}$, et à la substituer à l'étude de l'enchaînement et du déroulement des événements. Le caractère à la fois logique et mathématique des régularités statistiques les apparente à des lois universelles. On suppose que les relations observées se produisent constamment, c'est-à-dire aussi bien dans le passé que dans l'avenir, pourvu que toutes choses soient égales d'ailleurs, i.e. tant que les mêmes conditions sont réunies. A cette concession près à l'historicité de l'ordre humain, la prédiction du statisticien repose sur l'idée, implicite, que le temps est réversible, qu'on peut le parcourir, comme l'espace, dans les deux sens.

Cette conception du temps est au principe de l'épistémologie du déterminisme strict dont on trouve la formulation chez Laplace (Laplace 1986 [1814]: 32) ${ }^{11}$. Si l'on avait la connaissance complète de l'état initial du système, on pourrait tout prédire; cette connaissance n'est imparfaite qu'en raison de la faiblesse des moyens humains; elle est théoriquement possible, car les objets et les relations qui constituent l'état initial du système existent de tout temps et donc lui préexistent. Dans cette perspective, une relation statistique, dûment isolée, paraît d'autant plus vraie qu'elle semble plus universelle, c'est-à-dire, toutes choses égales d'ailleurs, moins dépendante des contextes historiques. L'histoire des individus, les orientations successives que le cours de leur vie a pu prendre, se réduisent comme toute histoire au déroulement chronologique d'une formule logique initiale. Les hasards de la vie ne sont que des accidents de parcours, des perturbations que la théorie néglige à juste titre pour construire ses modèles explicatifs; soit des hasards apparents, des illusions naïves, dont se berce le sens commun, et que la mise en évidence des régularités statistiques suffit à dissiper (comme le montre, entre cent exemples, le «choix» du conjoint); soit le résidu de l'analyse, ce que l'on ne parvient pas, provisoirement, à expliquer, mais qu'on expliquerait si l'on avait une connaissance suffisante des conditions initiales du système ${ }^{12}$.

${ }^{10}$ A. Fagot-Largeault, Les causes de la mort, histoire naturelle et facteurs de risque, Paris, Lyon, Vrin et Institut Interdisciplinaire d'Etudes Epistémologiques, 1989, p. 264. «Expliquer la mort» consiste au contraire «à dérouler la succession des événements qui la constituent, à déplier chaque événement pour y trouver une succession plus fine». $I d$, p. 260.

11 «Une intelligence qui, pour un instant donné, connaîtrait toutes les forces dont la nature est animée et la situation respective des êtres qui la composent (...) embrasserait dans la même formule les mouvements des plus grands corps de l'univers et ceux du plus léger atome: rien ne serait incertain pour elle, et l'avenir comme le passé serait présent à ses yeux. L'esprit humain offre, dans la perfection qu'il a su donner à l'Astronomie, une faible esquisse de cette intelligence ». P.-S. Laplace, Essai philosophique sur les probabilités, Paris, Christian Bourgois, 1986 [1814], pp. 32, 33.

12 «Nous dirons que l'évolution d'un système réel est déterministe si elle est réglée par une loi strictement causale. Les causes agissant sur le système considéré déterminent de façon univoque son évolution, sans aucune autre alternative possible: il doit suivre inéluctablement le chemin que ces causes lui prescrivent. Les mathématiciens énoncent ce principe en disant que les 'conditions initiales' du système déterminent de façon univoque son évolution au cours du temps. Le futur du système, mais aussi son passé, est une simple conséquence de ces conditions initiales ». G. Israel, La mathématisation du réel, Paris, Ed. du Seuil, 1996, p. 84.

Les révolutions de la physique contemporaine n'entraînent pas l'abandon du déterminisme, qui ne correspond pas seulement aux lois de la physique classique, mais, plus profondément, à l'idée de loi et à la conception légaliste et mathématique de la causalité. «[Galilée] découvre la nature mathématique, l'idée méthodique, il fraie la voie à l'infinité des découvreurs et des découvertes en physique. Il découvre, par opposition à la causalité universelle du monde sensible (...) ce qui depuis lors est appelé sans plus 'la loi de causalité', la 'forme a priori' du monde 'vrai' (idéalisé et mathématisé), la 'loi de 
Si le hasard n'existe pas, s'il n'est qu'une illusion, un effet de notre ignorance, le probable ne peut se définir que négativement, non comme ce qui pourrait se réaliser, mais comme écart par rapport à la réalité, comme approximation et défaut de certitude. C'est la position de Poincaré, pour qui «l'énoncé d'une loi quelconque est forcément incomplet. Cet énoncé devrait comprendre l'énumération de tous les antécédents en vertu desquels un conséquent donné pourra se produire (...) comme on ne sera jamais certain de n'avoir pas oublié quelque condition essentielle, on ne pourra pas dire: si telles ou telles conditions sont réalisées, tel phénomène se produira; on pourra dire seulement: si telles et telles conditions sont réalisées, il est probable que tel phénomène se produira à peu près (...) Pour toutes ces raisons, aucune loi particulière ne sera jamais qu'approchée et probable» (Poincaré 1927) ${ }^{13}$. Max Planck oppose les lois statistiques, «simplement probables », aux lois dynamiques, «nécessaires »:

[Cependant] la dynamique et la statistique ne devront pas être conçues comme se trouvant l'une vis-à-vis de l'autre dans un simple rapport de coordination et placées de ce fait sur un pied d'égalité. Les lois dynamiques satisfont en effet complètement à notre besoin de causalité et elles ont, à cause de cela, un certain caractère de simplicité; les lois statistiques, par contre, forment toujours un ensemble complexe qui ne se présente jamais comme quelque chose de définitif, car elles comportent toujours, à l'état latent, le problème de leur réduction à des lois dynamiques élémentaires (...) Aux yeux de tout le monde, il est en effet évident qu'une probabilité, fût-elle aussi petite qu'on le voudra, est séparée d'une impossibilité par un abîme infranchissable (....) Toutes les fois qu'on se trouvera en présence d'une loi, la première chose à se demander sera donc: cette loi estelle une loi statistique ou une loi dynamique? Il y a là un dualisme, et même un dualisme inévitable, dès lors que les lois statistiques ont droit de cité en physique, néanmoins bien des gens en ont été insatisfaits et ont cherché à le faire disparaître. Dans ce but, ils se sont résignés à nier l'existence de toute certitude et de toute impossibilité absolue et à n'admettre que des probabilités plus ou moins grandes. Selon eux, il n'y aurait plus de lois dynamiques dans la nature, mais seulement des lois statistiques et le concept de nécessité absolue serait à exclure de la physique. Contre cette opinion, qui est une erreur grossière et pernicieuse, on peut objecter que tous les phénomènes réversibles, sans exception, sont régis par des lois dynamiques (...) Mais il y a bien mieux encore: la physique, pas plus que n'importe quelle autre science, que cette science soit une science de la nature ou une science de l'esprit humain, ne peut se passer de la notion de loi absolue; sans cette notion,

la légalité exacte' [das Gesetz der exakten Gesetzlichkeit], d'après laquelle tout événement de la 'nature' (celle qui est idéalisée), doit obéir à des lois exactes. (...) Rien n'est changé (...) dans le monde des principes par la prétendue révolution philosophique que constituerait la critique de la 'loi classique de causalité' du côté de la nouvelle physique atomique. Car dans toute cette nouveauté, à mon avis, demeure ce qui est essentiel sur le plan des principes, à savoir: la nature mathématique en soi, la nature donnée dans des formules et à interpréter seulement dans des formules ». E. Husserl, $L a$ crise des sciences européennes et la phénoménologie transcendantale, op. cit., p. 61.

13 Strictement déterministe, Poincaré assimile le hasard à un caprice - improbable - de la Nature. Les savants croient que «toute loi pourra être remplacée par une autre plus approchée et plus probable, que cette loi nouvelle ne sera elle-même que provisoire, mais que le même mouvement pourra progresser indéfiniment, de sorte que la science en progressant possédera des lois de plus en plus probables, que l'approximation finira par différer aussi peu que l'on veut de l'exactitude et la probabilité de la certitude». Il faut «exiger, avant de conclure à la contingence des lois naturelles, que ce progrès ait un terme, que le savant finisse un jour par être arrêté dans sa recherche d'une approximation de plus en plus grande et qu'au-delà d'une certaine limite, il ne rencontre plus dans la Nature que le caprice.» $L a$ valeur de la science, op. cit., pp. 249 sq. 
la statistique elle-même ne fournirait que des résultats dénués de leur fondement le plus essentiel (Planck 1941: 61) ${ }^{14}$.

Cette définition négative de la probabilité dévalorise les sciences historiques; elle en fait des sciences incertaines, douteuses, des «sciences conjecturales » et donc des sciences mineures, de «petites sciences ${ }^{15}$. Rapportées à l'idéal de la loi universelle, les régularités historiques se retrouvent au niveau des règles empiriques et des recettes pratiques, faillibles et grevées d'exceptions, ce qui renvoie les sciences historiques du côté de l'essayisme et des «sagesses» (Meillet 1921: $15)^{16}$. Si l'idéal nomothétique joue un rôle aussi décisif dans la hiérarchisation des sciences, c'est sans doute parce que la pensée philosophique assimile la vérité des lois universelles (valables sans exception, en tout temps, en tout lieu et en toutes circonstances) à la vérité absolue. Autre manifestation de ce glissement de «l'universel» à «l'absolu», on passe de l'idée, juste, qu'un énoncé contextuel (non universel) n'est vrai que relativement au contexte dans les limites duquel il prétend être vrai, à l'idée qu'il n'est que relativement vrai, y compris dans les limites de ce contexte.

Une pratique plus réfléchie des statistiques conduit pourtant à des orientations épistémologiques très différentes. On constate en effet:

1. que des variables à expliquer, et que l'on est parvenu à expliquer sont aussi des variables explicatives (exemple classique: l'origine sociale «détermine» la réussite dans l'enseignement secondaire, la réussite dans l'enseignement secondaire prend le relais et «détermine» à son tour l'accès aux différentes études supérieures).

${ }^{14}$ Max Planck, Initiations à la physique, op. cit., pp. 61 sq. Plus radicalement, C. Bernard nie l'existence des lois statistiques: «J'avoue que je ne comprends pas pourquoi on appelle lois les résultats qu'on peut tirer de la statistique; car la loi scientifique, suivant moi, ne peut être fondée que sur une certitude et sur un déterminisme absolu et non sur une probabilité (...) Il faut reconnaître dans toute science deux classes de phénomènes, les uns dont la cause est actuellement déterminée, les autres dont la cause est encore indéterminée. Pour tous les phénomènes dont la cause est déterminée, la statistique n'a rien à faire; elle serait même absurde (...) on ne fait de la statistique que parce qu'on est dans l'impossibilité de faire autrement». C. Bernard, Introduction à la médecine expérimentale, op. cit., p. 194.

15 E. Renan, Souvenirs d'enfance et de jeunesse, Paris, Calmann-Lévy, s.d., p. XXX.

${ }^{16}$ Cf. A. Meillet: «Toutes les lois générales qu'on a posées, toutes celles dont cette recherche, à peine entamée, réserve encore la découverte, ont cependant un défaut: elles énoncent des possibilités, non des nécessités. Ainsi la loi relative à la débilité caractéristique de l'articulation des consonnes intervocaliques n'empêche pas les consonnes de subsister entre voyelles durant un temps illimité dans certaines langues (...) Les lois de la phonétique ou de la morphologie générale historique ne suffisent donc à expliquer aucun fait; elles énoncent des conditions constantes qui règlent le développement des faits linguistiques; mais même si l'on parvenait à les déterminer d'une manière complète et de tout point exacte, on ne saurait pour cela prévoir aucune évolution future, ce qui est la marque d'une connaissance incomplète». Pour expliquer la persistance et la multiplicité des exceptions qu'il énumère, Meillet se réfère pourtant à une conception moins légaliste et plus historique de la probabilité; la réalisation des prédictions de la phonétique dépend en effet de la réunion, et donc de la rencontre, des «conditions variables qui permettent ou provoquent la réalisation des possibilités ainsi reconnues». A. Meillet, Linguistique historique et linguistique générale, Paris, Champion, 1921, p. 15. 
2. que, variante aggravée du cas précédent, la variable expliquée explique aussi la variable explicative, l'effet retentit sur la cause devenue conséquence, la renforce quand elle redevient cause, et ainsi de suite. On est en présence d'un processus cumulatif, d'un cercle «vicieux» ou «vertueux». Les relations statistiques que l'on a établies recouvrent donc un enchaînement de causes et d'effets.

3. que les variables explicatives sont elles-mêmes en relation les unes avec les autres (ainsi le niveau de revenu s'élève en même temps que le niveau de diplôme; la profession exercée dépend du niveau de diplôme et détermine le niveau de revenu; ou encore les différents types d'études supérieures sont très inégalement féminisés, ont des recrutements sociaux très différents). Il faut donc admettre que plusieurs variables concourent à produire le même effet, que tout ce que l'on peut faire est de mesurer et de hiérarchiser leurs effets respectifs; il faut renoncer au principe qui veut «qu'à un même effet corresponde toujours une même cause ». Par ailleurs, la présence de la propriété A ne détermine pas toujours, dans tous les cas, c'est-à-dire pour chaque individu l'effet B; une variable n'est donc pas à proprement parler une cause, mais un facteur de risque (ou de chance). De même que l'effet attendu peut ne pas se produire, l'inattendu, l'improbable peut se réaliser.

Probabiliste dans son principe, l'étude statistique invite à rompre avec le déterminisme absolu et à reconnaître l'existence du hasard. C'est la position de Cournot, pour qui l'idée du hasard «est la clé de la statistique, et donne un sens incontestable à ce que l'on a appelé la philosophie de l'histoire, à ce que nous aimerions mieux appeler l'étiologie historique, en entendant par là l'analyse et la discussion des causes ou des enchaînements de causes qui ont concouru à amener les événements dont l'histoire offre le tableau (...) Le fait naturel ainsi établi ou constaté consiste dans l'indépendance mutuelle de plusieurs séries de causes et d'effets qui concourent accidentellement à produire tel ou tel phénomène, à amener telle ou telle rencontre, à déterminer tel événement, lequel, pour cette raison est qualifié de fortuit» (Cournot 1973 [1872]) ${ }^{17}$. Les régularités statistiques demandent elles-mêmes à être expliquées. On peut sans doute essayer d'aller toujours plus loin dans l'analyse statistique, d'entrer dans le détail en utilisant des catégories de plus en plus fines. Pour expliquer, par exemple, pourquoi il n'est pas exclu que des filles d'ouvrier entrent à l'ENS, section sciences, voire à l'Ecole polytechnique, on montrera que celles-ci sont porteuses d'avantages particuliers, qui les distinguent des autres filles d'ouvrier, et qui compensent les handicaps liés au sexe et à l'origine sociale. On s'appuie ainsi sur la pluralité des causes, ou sur ce que C. Bernard appelait le «déterminisme complexe», pour ramener l'exception à la règle, réduire la part du hasard et revenir au déterminisme strict. Mais, si loin que l'on aille dans cette direction, on laissera toujours échapper la genèse des phénomènes, le déroulement chronologique des enchaînements dont ils sont

17 A. A. Cournot, Considérations sur la marche des idées et des événements dans les temps modernes, op. cit. Sur la «complémentarité du déterminisme et du hasard» chez Cournot, cf. T. Martin, «La valeur objective du calcul des probabilités selon Cournot», Mathématiques et informatique en sciences humaines, $\mathrm{n}^{\circ} 127,1994$, pp. 5-17. 
issus; à force de rapprocher l'analyse statistique de l'étude de cas en utilisant des catégories sans cesse plus fines, on perd l'efficacité simplificatrice de la première sans atteindre la capacité explicative de la seconde.

Si l'on admet que le hasard existe, qu'il n'est pas seulement la mesure de notre ignorance, il faut renoncer à tout prédire; faute de s'être réalisé au moment de la prédiction, ce qu'il faudrait savoir pour prédire peut n'être pas connaissable, et l'on ne peut pas savoir s'il le sera. C'est seulement en reconstituant l'histoire des rencontres entre des séries causales plus ou moins indépendantes que l'on peut expliquer comment ce qui était un possible parmi d'autres s'est réalisé, et comment les autres possibles, possibles latéraux, possibles concurrents ne se sont pas réalisés; il faut également retracer cette histoire pour déterminer les effets de la réalisation du possible «sélectionné », les possibilités qu'elle laisse ouvertes, celles qu'elle ouvre, celles qu'elle interdit, ce qu'elle rend impossible. La «construction de l'irréel » ${ }^{18}$ (Aron 1962: 194) est le seul moyen d'expliquer pourquoi tel accident est survenu, et d'en apprécier la portée, de déterminer ses conséquences, la nature et l'ampleur des changements qu'on aurait observés si tel événement n'avait pas eu lieu. C'est le paradigme du «nez de Cléopâtre» (Pascal, parieur, admet le hasard, avant Cournot), et le principe d'une histoire contrefactuelle dont s'amuse le réalisme du bon sens («avec des si...»). Le raisonnement contrefactuel est au principe de la simulation, qui consiste à changer un ou plusieurs éléments des conditions initiales (paramètres ou variables exogènes d'un modèle en économie appliquée). Le sociologue statisticien ne cesse de comparer ce qui est à ce qui aurait pu être. S'il s'en tenait aux variations de fait, constatées par l'enquête, il ne pourrait ni tester ni interpréter les relations entre les variables; il faut qu'il compare les valeurs constatées aux valeurs que les variables auraient pu prendre (c'est ce que l'on fait chaque fois que l'on calcule un $\chi 2$, on compare les effectifs observés aux effectifs théoriques). Mais c'est en dehors du temps historique que ces «expériences de pensée » conduisent à imaginer un autre état du monde. D'où les contradictions dans lesquelles la résistance du passé enferme le raisonnement contrefactuel quand on essaie de l'étendre à l'histoire. Ou bien l'on se contente de changer un nombre réduit d'éléments dans les conditions initiales du modèle, et l'on reste dans le vague; ou bien on va le plus loin possible dans l'inventaire et dans l'explicitation des changements possibles, et l'on risque de tomber dans l'absurde, c'est-à-dire d'imaginer un monde incapable de s'insérer dans le monde qui s'est réalisé ${ }^{19}$ (Elster 1978, Lewis 1976).

La construction de l'irréel pose le problème de la détermination du moment où l'on revient dans le passé. Si l'on revient en amont de l'événement auquel on s'intéresse, à un moment qui précède sa réalisation et la non réalisation des possibles concurrents, on fait non pas de la rétrodiction, mais de la prédiction, et de la prédiction dans le passé. A la différence des contemporains, on connaît la suite des

18 «La procédure par laquelle on parvient à une causalité historique (...) comporte comme l'une de ses démarches essentielles la construction de ce qui se serait passé si l'un des antécédents ne s'était pas produit ou avait été autre qu'il n'a été.» R. Aron, Les grandes doctrines de sociologie historique, op. cit., t. II, p. 194.

${ }^{19}$ Cf. J. Elster, Logic and Society: contradictions and possible worlds, New York, Wiley, 1978; D. K. Lewis, Counterfactuals, Cambridge, Mass., Harvard University Press, 1976. 
événements, et on ne peut l'ignorer, on ne peut pas faire comme si on ne la connaissait pas. On prédit alors à coup sûr, sans démenti possible. La prédiction dans le passé ne laisse aucune place au hasard. Savoir la conclusion, le «fin mot», de l'histoire entraîne un biais de sélection; on ne retient que les faits qui ont concouru à la réalisation de ce qui s'est effectivement produit, on ne pense pas à ceux qui concouraient à la réalisation des possibles latéraux. La connaissance du terme final du processus fait ainsi oublier que ce qui fut aurait pu ne pas être, que l'histoire aurait pu tourner différemment, qu'on aurait pu arriver au même dénouement par d'autres chemins. Comme on connaît aussi le début de l'histoire, que l'on tient les deux bouts de la chaîne, on a et on donne encore plus l'impression que tout s'enchaîne, que les choses ne pouvaient pas tourner autrement. La logique imperturbable (i.e. insensible aux perturbations) avec laquelle ce genre d'explication ex post enchaîne les événements fait évidemment penser à ces débats moliéresques dont les participants reconstituent la genèse des événements avec d'autant plus d'assurance qu'ils les ont moins vu venir : «et voilà pourquoi votre fille est muette». Par ailleurs, comment déterminer la date de cet état antérieur, c'est-à-dire la distance qui le sépare de l'événement considéré? Si on revient trop en amont, si l'on remonte trop loin dans le passé, l'éventail des possibles est trop large et les chances de réalisation de ce qui s'est finalement produit sont quasi nulles; si l'on revient trop près, la plupart des possibles latéraux ont déjà disparu, les jeux sont faits (Popper 1996 [1982]: 177) ${ }^{20}$.

Pour expliquer «ce qui s'est passé», il faut donc se placer au moment de la survenue de l'événement considéré et remonter vers le passé, en reconstituant les processus qui ont concouru à la production de ce qui s'est effectivement produit, et qui ont empêché que les autres issues, que les autres possibles se réalisent. Aux prédictions de l'étude statistique, il faut associer la rétrodiction, entendue comme la reconstitution détaillée, en remontant le temps et des effets aux causes, des processus, de «séquences d'événements» toujours plus microscopiques,

20 «Imaginons que nous ayons attaché une bande de film à un certain état instantané donné du monde, c'est-à-dire, à une tranche de temps donnée du monde, et que nous utilisions cette bande de film pour décrire toutes les tranches de temps passées et futures du monde, du mieux que nous le pouvons (...) Nous supposerons, comme premier pas ou première approximation, que la bande de film que nous avons attachée à la tranche de temps donnée est laplacienne ou déterministe, ou, en d'autres termes, qu'elle décrit un univers en bloc et déterministe - celui qui est déterminé par l'état instantané donné ou la tranche de temps; car nous savons qu'un état instantané, ou une tranche de temps, est suffisant, selon Laplace, pour déterminer tous les états ou tranches de temps passés et futurs d'un univers déterministe. Nous supposons que chacun des états instantanés ou tranche de temps de l'univers déterministe est décrit par une des photographies dont la bande du film est composée (...) Dire que notre propre univers - l'univers réel - est non déterministe est insinuer que les bandes laplaciennes de films que nous avons attachées aux différentes tranches de temps ne décrivent pas exactement notre propre univers (...) Sans doute, les quelques premières photographies qui viennent avant et après la tranche de temps réelle à laquelle le film est attaché seront très semblables aux états ou aux tranches de temps réels qu'elles sont supposées décrire: nous savons cela à partir du succès de la physique classique. Mais des différences mineures vont s'accumuler si nous nous écartons de plus en plus de la tranche de temps du monde réel à laquelle le film est attaché; et si nous nous éloignons assez de cette façon, les photographies deviendront alors inutiles aux fins de la prédiction ». K. Popper, La théorie quantique et le schisme en physique, Hermann, Paris, 1996, trad. de The Postscript to the Logic of Scientific Discovery, III, Quantum theory and the Schism in Physics, 1992, pp. 177 sq. 
jusqu'aux nano-histoires que l'histoire suggérée par les relations statistiques, qui en donnent seulement le début et la fin, l'entrée et la sortie, invite à découvrir. On ne peut retracer complètement «l'ordre des causes» sans faire l'inventaire rétrospectif des «faits de hasard», des occasions (même origine que «accident» ou que «cas»), des collisions, des chocs, bref sans reconstituer la succession des rencontres plus ou moins probables entre des séries causales indépendantes qui déterminent l'orientation, la tournure que prend une histoire. La construction de l'irréel risque sans doute de s'aventurer dans le domaine de la fiction, de revenir à la littérature. «L'histoire est un roman qui a été; le roman est de l'histoire qui aurait pu être» (Goncourt 1989) ${ }^{21}$. Mais on peut mettre à profit la nécessité d'élaborer et de comparer des scénarios, commune à l'enquête scientifique, à l'enquête policière et au roman policier. Il se peut que le coupable réel soit le moins probable, en tout cas moins probable que le coupable supposé, celui que tout accuse, ou paraît accuser. Pour entretenir la croyance spontanée en son innocence, le coupable procède à une reconstitution de l'irréel. Inversement, l'enquêteur procède à la reconstitution d'une réalité, invraisemblable à l'origine, qui ne paraît aller de soi (bon sang mais c'est bien sûr!) que lorsque «tous les fils ont été noués », c'est-à-dire une fois que les relations entre les faits contrariants ont été établies. Pour imaginer ce qui serait arrivé si la vie de leurs personnages avait pris un autre cours, le romancier ou le cinéaste se fondent sur ce qu'ils savent d'autres cas, à la fois différents et similaires, de variantes divergentes de la même famille. Le sociologue qui compare des études de cas, en sélectionnant des histoires qui se ressemblent à l'origine, et qui ont tourné différemment, procède de la même manière. Mais la fiction dispense d'imaginer l'ensemble complet des possibles, l'enchaînement des bifurcations, de rang deux, trois, etc.; elle se borne à envisager les éventualités les moins invraisemblables, c'est-à-dire les plus conformes aux attentes du sens commun (qui ne veut être surpris que dans ses propres limites), et, parmi ces possibilités, elle choisit de faire se réaliser celles qui sont le plus capables de procurer l'illusion de la réalité. C'est précisément à ces obligations - inventaire exhaustif des possibles, hiérarchisation et sélection des possibles en fonction de leurs probabilités de réalisation - que la construction scientifique de l'irréel doit satisfaire.

\section{LES ÉTUDES DE CAS}

L'enquête statistique demande donc à être combinée avec des techniques d'investigation qualitatives et narratives, comme l'observation directe et l'interview. Les chroniques individuelles, les biographies sont pour le sociologue l'équivalent des études de cas cliniques en physiopathologie; le couple enquête par questionnaire / entretien biographique ressemble beaucoup à l'association de la statistique épidémiologique et de l'étude de cas clinique décrite par A. Fagot-Largeault (1989: 275) $)^{22}$. L'entretien - «l'interrogatoire »- sociologique permet de retracer

${ }^{21}$ J. et E. de Goncourt, Journal, Paris, Laffont, 1989, t. 1, p. 750.

22 «Ainsi l'explication causale [de la mort chez les malades du croup] chez Louis oscille entre deux pôles: d'un côté, la reconstitution aussi détaillée que possible de la 'marche', ou de l'histoire du 
les hasards des rencontres entre des séries causales indépendantes, notamment entre des prédispositions liées à des antécédents (la "génétique sociale», équivalent du «terrain») et l'offre d'événements, plus ou moins probables, caractéristique d'un milieu, c'est-à-dire d'un environnement social et d'un contexte historique particuliers.

C'est ce que fait voir une enquête auprès de végétariens plus ou moins intransigeants, adhérents d'une coopérative de vente de produits biologiques ${ }^{23}$. L'étude statistique, par questionnaire, montre que ceux-ci présentent un ensemble systématique de traits par lesquels ils se distinguent de la population dont ils font partie: le végétarisme se rencontre plus souvent chez les femmes, dans les professions intellectuelles et chez les titulaires d'un diplôme élevé, et, parmi les diplômés et les membres des professions intermédiaires ou supérieures, plus souvent chez ceux qui ont un revenu inférieur au revenu moyen dans ces catégories ${ }^{24}$. Surtout, la plupart des enquêtés ont eu un «destin social» improbable. Si l'on compare les coopératrices qui ont répondu à l'enquête à l'ensemble des femmes de même origine, et de même génération, on constate que leur ascension sociale est en général très supérieure, en particulier pour celles qui sont issues des classes populaires et des couches inférieures des classes moyennes ${ }^{25}$; leurs trajectoires scolaires ne sont pas moins exceptionnelles que leurs trajectoires sociales.

On se trouve ainsi en présence d'une correspondance, troublante et suggestive, entre l'écart par rapport aux pratiques alimentaires standard et l'écart par rapport à la trajectoire ordinaire, la plus probable. L'enquête fait apparaître un ensemble de conditions favorables à la conversion au mode d'alimentation et à la règle de vie particuliers qu'on observe chez les enquêtés. Ces conditions sont nécessaires, puisque, inversement, cette conversion se produit moins souvent quand elles ne sont pas présentes. Mais elles ne sont pas suffisantes. Tant que l'on se place au départ, on constate que les trajectoires sociales suivies par les enquêtés se sont beaucoup écartées de celles qu'ils auraient dû suivre, c'est-à-dire des trajectoires effectivement suivies par la majorité des individus de la même génération ayant la même origine; mais si l'on se place à l'arrivée, on voit que les enquêtés partagent

phénomène, de l'autre, l'évaluation comparative des fréquences (...) Si le contenu de l'explication s'est enrichi (...), la démarche explicative (...) se développe toujours à la fois sur le mode épidémiologique et sur celui de la chronique (...). L'histoire de la progression du bacille elle-même ne fait que manifester une séquence plus microscopique d'événements $(. .$.$) . «On ne connaît pas de limite à ce$ processus d'explication progressive de la séquence d'événements qu'au niveau tout à fait macroscopique on appelle, d'un mot, le croup ». A. Fagot-Largeault, Les causes de la mort, op . cit., pp. 275, 276.

${ }^{23}$ Enquête réalisée dans le cadre du Laboratoire de recherche sur la consommation de l'INRA; on a par ailleurs observé pendant une semaine le fonctionnement de la coopérative, assisté à des réunions, étudié les relations entre les responsables, les salariés et les adhérents, et réalisé à cette occasion des entretiens approfondis de militants et de sympathisants actifs.

${ }^{24}$ La méthode est la même que celle qui consiste à vérifier la représentativité d'un échantillon par rapport à une population de référence, mais l'objectif est inverse. Au lieu de s'assurer de la représentativité du groupe des enquêtés, on se propose au contraire de déterminer sous quels rapports et à quel degré celui-ci se distingue de l'ensemble des consommateurs.

$253,9 \%$ seulement des enquêtées filles d'ouvrier sont elles-mêmes ouvrières, contre $34,1 \%$ de l'ensemble des filles d'ouvrier; $24,4 \%$ sont employées, contre 43,5\%. Inversement, $16,7 \%$ sont institutrices, contre $2,3 \%$ et $7,7 \%$ professeurs, contre $0,8 \%$. 
avec la majorité des individus qui appartiennent aux mêmes catégories sociales qu'eux le destin non-conforme qui les distingue de l'ensemble de la population. Les écarts qu'on relève sur le tableau des recrutements se réduisent et parfois même s'inversent par rapport à ceux qui figurent sur le tableau des destins. Ainsi les enquêtées professeurs sont moins nombreuses, proportionnellement, que l'ensemble des femmes professeurs à être filles d'ouvrier $(10,2 \%$ contre $13,9 \%)$; de même, la proportion de filles d'ouvrier est plus faible parmi les institutrices végétariennes que parmi l'ensemble des institutrices (16,5\% contre 18,5\%). Tout ce que l'on peut dire, c'est donc que les individus dont la trajectoire sociale s'est écartée de la trajectoire qu'ils auraient dû suivre ont davantage de chances de développer un mode de vie déviant. En dressant l'inventaire des surreprésentations par lesquelles notre population d'enquêtés se distingue de la population française, nous avons défini, à l'intérieur de l'ensemble de celle-ci, un sous-ensemble qu'on pourrait appeler population à risque, dont notre population particulière fait partie, mais dont elle se distingue dans la mesure où cette sous-population à risque est constituée dans sa grande majorité de "porteurs sains », alors que nos enquêtés ont développé, eux, l'anomalie (i.e. l'écart par rapport au comportement standard) dont nous essayons de trouver les causes.

Pour expliquer pourquoi les enquêtés se sont mis à refuser l'alimentation communément admise, il faut donc partir à la recherche de causes supplémentaires. Comme on l'a vu, on n'irait pas très loin en continuant dans la même voie, en complétant le tableau statistique des prédispositions par l'ajout de nouvelles caractéristiques distinctives à la liste des variables explicatives et en entrant plus avant dans la combinaison de ces caractéristiques. Ces causes supplémentaires rendent sans doute plus probable l'apparition du non-conformisme; l'idée sousjacente est qu'à force d'ajouter des causes nécessaires, on finira bien par réunir l'ensemble complet (ou presque) des causes suffisantes. Mais le sous-ensemble considéré se réduit à chaque ajout, de sorte que cette méthode trouve vite ses limites. Il faut donc passer aux études de cas proprement dites, qui permettent, elles, de reconstituer la succession, si possible l'enchaînement, des événements particuliers qui sont arrivés aux enquêtés. Les récits autobiographiques permettent de pénétrer rétrospectivement dans les milieux où ils ont vécu, de reconstituer ce qui s'est passé dans tel village, tel quartier, telle famille, telle école, etc. On entre ainsi dans le détail des mécanismes sociaux de marquage, de mise à l'écart, d'exclusion, qui entraînent une intégration manquée et défectueuse, et que signalent, mais recouvrent les écarts statistiques à la moyenne. Cette microsociologie est aussi une microhistoire. L'entretien biographique permet de changer d'échelle temporelle, de passer du résumé, du survol d'une longue période à la reconstitution minutieuse d'une journée, de découper des séquences aussi courtes et aussi denses en événements que nécessaire. On peut alors isoler des épisodes critiques, des interventions, des rencontres décisives; on voit apparaître des agents qui ont joué un rôle déterminant dans la vie des enquêtés. On observe, sous le plus fort grossissement possible, les moments où celle-ci a bifurqué, les enchaînements d'événements et les concours de circonstances, plus ou moins accidentels, plus ou moins imprévus, qui ont fait dévier les enquêtés de la trajectoire qu'ils auraient dû suivre. On voit ainsi, et on s'explique, comment le possible le plus probable a pu ne pas se réaliser, comment des causes aussi déterminantes que l'origine sociale ou l'échec scolaire n'ont pas produit leurs effets, comment de petits événements, 
à première vue insignifiants, amorcent une succession de mécanismes cumulatifs et ont ainsi des conséquences «disproportionnées », comment des déviations très faibles, consécutives à des chocs minimes, à de légers coups de pouce entraînent à l'arrivée, tels le clinamen de Lucrèce, des écarts importants. On tourne ainsi le dos aux principes sur lesquels repose la conception légaliste et purement logique de la relation causale (tels qu'on les trouve chez Durkheim); on opte, à l'inverse, pour la pluralité des causes et pour la disproportion entre la cause et l'effet.

Dans toutes les sciences le recours aux études de cas pose un ensemble de problèmes identiques. Une première difficulté tient au choix des cas, choix décisif puisqu'en dépend le degré auquel ils sont représentatifs. Qu'on s'abandonne trop au hasard des rencontres et aux occasions du terrain, ou, à l'inverse, que l'on choisisse délibérément les enquêtés pour leur caractère outré qui les apparente à un type idéal (ou aussi bien à un personnage de roman), parce qu'ils semblent personnifier l'idée préconçue que l'on a de la pratique que l'on étudie (par exemple «incarner» le végétarien et donc le végétarisme), on risque de généraliser abusivement, de prendre des cas singuliers pour des cas exemplaires et typiques. Autre choix décisif, la sélection des traits pertinents, «l'identification, dans les cas individuels, des événements significatifs » (Fagot-Largeault 1989: 275) ${ }^{26}$ qui permettent de définir des histoires-types; elle peut, elle aussi, être biaisée par les a priori de l'enquête, et elle dépend du rapport que les enquêtés entretiennent avec leur passé et avec l'examen auquel ils sont soumis, avec la singularité qui les constitue en cas et pour laquelle on les questionne; outre qu'ils tendent, tels le sociologue ou le médecin déterministe, à oublier les possibles qui ne se sont pas réalisés, la situation d'entretien les incite, on le sait, à idéaliser et à rationaliser leur histoire, à oublier ou à passer sous silence les épisodes qui ne coïncident pas avec l'idée qu'ils se font et qu'ils entendent donner d'eux-mêmes.

Dans les sciences de l'homme, les études de cas présentent des risques supplémentaires. Le principal est sans doute l'illusion qui fait croire que les faits sociaux, en tant que faits «humains », peuvent faire l'objet d'une compréhension immédiate, par intuition, par empathie, en se mettant par la pensée à la place de l'autre. Cette croyance est au principe de l'opposition entre «comprendre » et «expliquer», qui fait partie depuis Max Weber de la tradition sociologique. M. Weber distingue entre «l'adéquation de sens, c'est-à-dire un déroulement conforme à notre manière de penser et de sentir» (Aktuelles Verstehen) et «l'adéquation causale» (Verklärendes Verstehen) ${ }^{27}$. Dans la mesure où l'idée de

26 A. Fagot-Largeault, Les causes de la mort, op. cit., p. 275.

${ }^{27}$ Pour M. Weber, «Verstehen désigne deux opérations distinctes, Aktuelles Verstehen et Verklärendes Verstehen, la compréhension immédiate que l'on a d'un acte comme celui du bûcheron en train d'abattre un arbre et la compréhension explicative (qui nous renvoie aux motifs) comme le fait que le bûcheron travaille pour gagner sa vie». La conduite du bûcheron «est pour nous compréhensible (et la logique de son enchaînement nous renvoie à la notion de richtig = correct, juste). Mais il est des séquences d'événements dont nous pouvons seulement dire qu'elles se déroulent avec régularité (Regel) et selon une loi statistique. Il n'y a plus d'adéquation de sens, mais une relation qui servira à l'explication sans compréhension». F. A. Isambert, «Max Weber désenchanté», L'Année sociologique, 1993, 43, pp. 357-397. «En donnant à la sociologie pour tâche de 'comprendre' (verstehen) Weber se situe d'emblée dans le débat de la pensée allemande sur la science. Celui-ci, en opposant de diverses manières sciences humaines et sciences de la nature, avait tendu à faire de la 'compréhension' 
compréhension participe d'un égocentrisme qui est la forme spontanée et primitive de l'ethnocentrisme, elle constitue pourtant un obstacle permanent à la prise de distance et au sentiment d'étrangeté par rapport au «cela va de soi» du milieu dont on est l'indigène, qui sont le point de départ et la condition de la réflexion sociologique, ethnologique ou historique. En se mettant à la place de l'autre, on en fait son semblable et l'on ne peut voir chez lui, et plus généralement dans l'altérité des cultures, des classes ou des époques différentes que des similitudes qui paraissent évidentes parce qu'elles sont triviales ${ }^{28}$.

L'illusion de la compréhension immédiate réactive les idées fusionnelles d'empathie, de compassion et de communion; elle relève du «principe de participation» dans lequel L. Lévy-Bruhl voyait une caractéristique essentielle de la mentalité primitive, à la fois prélogique et mystique (Lévy-Bruhl 1910: 76 sq.) ${ }^{29}$. Elle renforce la «liaison intime des études historiques avec la politique et la morale» (Renan 1876) $)^{30}$ et la dérive édifiante dont la sociologie critique est menacée. Plus on croit comprendre ce que l'on ne sait pas expliquer, et moins on a besoin de préciser le sens des termes que l'on emploie, de donner un sens nouveau et particulier aux mots du langage ordinaire, de créer des mots nouveaux, bref de se doter d'un langage savant spécialisé; plus les «mots primitifs» dont parle C. Bernard semblent suffire, plus ils viennent spontanément à l'esprit et sous la plume ${ }^{31}$. L'illusion de la compréhension immédiate contribue ainsi à renvoyer les sciences de l'homme du côté de la littérature. Elle pousse à préférer et à rechercher les mots et les procédés expressifs, capables de susciter et de réactiver l'impression d'empathie et d'amener le lecteur à s'identifier aux personnages et aux situations qu'on lui présente. Le langage littéraire paraît aller de soi, et semble même techniquement tout indiqué; son pouvoir d'évocation en fait le langage

(Verstehen) le propre des premières et de 'l'explication' (Erklärung) l'opération caractérisant les secondes. La formule décisive avait été énoncée par Dilthey (Gesammelte Schriften, t. V, p. 143): 'Die Natur erklären wir, das Seelenleben verstehen wir'». F.A. Isambert, «Sens, compréhension et valeur chez Max Weber», Ethique et Pratiques symboliques, Cahier n³, EHESS, CNRS, 1986.

${ }^{28}$ C'est sans doute de cette illusion que procède l'idéologie identitaire qui sévit actuellement dans nos disciplines, selon laquelle le semblable serait seul habilité à étudier le semblable.

${ }^{29}$ «Il serait difficile de donner, dès à présent, un énoncé abstrait de cette loi [la «loi de participation»] (...) Je dirais que, dans les représentations collectives de la pensée primitive, les objets, les êtres, les phénomènes peuvent être, d'une façon incompréhensible pour nous, à la fois eux-mêmes et autre chose qu'eux-mêmes. D'une façon non moins incompréhensible, ils émettent et ils reçoivent des forces, des vertus, des qualités, des actions mystiques, qui se font sentir hors d'eux, sans cesser d'être où elles sont. En d'autres termes, pour cette mentalité, l'opposition entre l'un et le plusieurs, le même et l'autre, etc., n'impose pas la nécessité d'affirmer l'un des termes si l'on nie l'autre, ou réciproquement. Elle n'a qu'un intérêt secondaire. Parfois, elle est aperçue; souvent aussi, elle ne l'est pas. Souvent elle s'efface devant une communauté mystique d'essence entre des êtres qui cependant, pour notre pensée, ne sauraient être confondus sans absurdité. Par exemple, 'les Trumai (tribu du nord du Brésil) disent qu'ils sont des animaux aquatiques. - Les Bororó se vantent d'être des araras (perroquets) rouges' (...) ce n'est pas un nom qu'ils se donnent, ce n'est pas une parenté qu'ils proclament. Ce qu'ils veulent faire entendre, c'est une identité essentielle.» L. Lévy-Bruhl, Les fonctions mentales dans les sociétés inférieures, Paris, Alcan, 1910, pp. 76 sq.

${ }^{30}$ E. Renan, Dialogues et fragments philosophiques, op. cit., p. 155.

${ }^{31}$ C. Bernard, «Définition de la vie» [1875], in La science expérimentale, op. cit. «Le mot vie est dans ce cas. Tout le monde s'entend quand on parle de la vie et de la mort». 
naturel de la sociologie. Comme le romancier, le sociologue, l'ethnologue ou l'historien s'essaient à donner l'illusion de la réalité; dès lors que l'on croit qu'il suffit de comprendre, on croit qu'il suffit de peindre pour expliquer ${ }^{32}$.

Les interviews, qui sont un des instruments essentiels des études de cas en sociologie, et qui sont aussi une technique d'enquête et un genre d'écriture que le sociologue partage avec le journaliste, sont particulièrement sujets à l'illusion de la compréhension immédiate. Leur présentation conserve la trace de la sympathie, apparente ou réelle, qui permet à l'enquêteur d'obtenir la confiance et les «confidences » de l'interviewé. En jouant sur les interactions du langage populaire et de la langue savante (dans la tradition du roman réaliste), on obtient un «effet de réel » qui pousse le lecteur à s'identifier aux enquêtés. C'est le cas de l'interviewtémoignage, dont le montage privilégie les extraits les plus évocateurs, ceux qui attirent et stimulent le plus la sympathie du lecteur et lui donnent au plus haut point l'impression d'être à la place de l'interviewé et de le comprendre de l'intérieur; le pacte de lecture qu'ils instaurent est une communion de subjectivités. Il faut sans doute distinguer entre l'interview-témoignage et l'interview critique, où le sociologue, se tenant à distance, essaie de dégager le sens objectif du discours de l'enquêté, en fonction d'une batterie pré-établie d'indicateurs symptomatiques; du moins faudrait-il pouvoir le faire. En pratique la présentation des entretiens mélange trop souvent le témoignage et l'analyse, ou plutôt ce qui en tient lieu, la paraphrase en langue savante de fragments de discours indigènes choisis pour leur caractère exemplaire et leur capacité à servir d'illustration à un parti-pris interprétatif. L'interview constitue la pièce maîtresse d'une sociologie populiste de la misère issue d'une sociologie légitimiste de la domination; la sociologie du cœur est ainsi devenue le dernier avatar de la sociologie à l'estomac.

La combinaison de l'enquête statistique et de l'étude de cas est donc particulièrement difficile et risquée dans les sciences de l'homme. Elle y est pourtant indispensable, à la fois parce que les deux démarches se renforcent mutuellement et parce que l'explication des phénomènes la requiert. L'étude statistique n'explique pas comment le plus probable peut ne pas se réaliser, ni comment le moins probable se réalise; mais le raisonnement probabiliste empêche de confondre le vrai et le réel, d'oublier que ce qui s'est réalisé ne fut jamais qu'un possible, plus ou moins probable, parmi d'autres. Les études de cas permettent de reconstituer les processus au terme desquels une possibilité a fini par se réaliser à l'exclusion d'autres possibilités concurrentes. On voit ainsi que les trajectoires sociales ne sont pas des «destins», mais le résultat de la rencontre entre un héritage et un environnement, entre des propriétés sociales (le terrain d'origine et ses modifications successives) et le cours du monde historique (les changements qui affectent le milieu d'origine des enquêtés et les milieux successifs qu'ils traversent), c'està-dire entre des séries causales dont l'indépendance ne se laisse pas réduire.

${ }^{32}$ Cf. supra, chap. III, 3. 